\title{
Genomic Cloning and Sequence Analysis of Trypanosoma brucei rhodesiense Gene Encoding Putative N-glycosylation Enzyme
}

\author{
Waren Navarra Baticados', Noboru Inoue², Chihiro Sugimoto³, Hideyuki Nagasawa² \& Abigail Morales \\ Baticados ${ }^{1}$
}

\begin{abstract}
Background: Trypanosoma brucei rhodesiense is a haemoflagellate parasite of zoonotic significance. Aside from its public health importance, this parasite subspecies gained notoriety because of their effective system to circumvent the immune response of vertebrate host. The parasite cell surface is covered with millions of VSG dimers, which serve as an almost infinite repertoire of biomolecules needed for evasion of host immune system. Around two decades ago, it was resolved that all trypanosome VSG is associated with one or more $\mathrm{N}$-linked oligosaccharides, with a range of structures including high mannose and complex types. This complex process of protein modification known as N-linked glycosylation is catalyzed by oligosaccharyl transferase (OST). In general, the incorporation of glycan structures can alter protein's antigenic properties and recently it was established that glycan molecules associated with VSG were found to be important in several aspects of trypanosome-host interaction, especially during parasite evasion of the host defense mechanisms. Therefore, our major interest is to clone and characterize the trypanosome OST.

Material, Methods and Results: The template genomic DNA for PCR amplification was extracted as described previously. In an attempt to clone Trypanosoma brucei rhodesiense putative oligosaccharyl transferase, an amplicon of $\sim 2000$ bp was obtained having an open reading frame of $2057 \mathrm{bp}$ and deduced primary structure composed of 685 amino acid residues (TbrOST II). Comparison of TbrOST II ORF with annotated putative oligosaccharyl transferase in the genome of other organisms revealed sequence identity to other kinetoplastid. TbrOST II had high nucleotide (Ns) and amino acid (As) sequence similarity with the genomes of T. brucei gambiense (Ns:99\%; As:78\%) and T. brucei (Ns:95-98\%; As:77\%-98\%). There was also significant nucleotide and amino acid sequence identity in the genomes of T. cruzi (Ns:74\%; As:63\%), Leishmania infantum (Ns:70-83\%; As:46-57\%), L. braziliensis (Ns:69-81\%; As:46-55\%) and L. major (Ns:69-80\%; As:46-57\%). Sequence similarity (71-77\%) from other origins was also exhibited. The nucleotide sequence alignments and analysis were performed using the Oxford University Mac Vector 6.5 sequence analysis software and CLC Workbench 5.6 software.

Discussion: The nucleotide BLAST results indicate that sequence identity is higher between species of the same genus rather than of the same family. It is known that T. brucei, T. gambiense and T. rhodesiense are members of the Brucei-complex or Brucei group. Although T. brucei brucei has more similarities with T. brucei rhodesiense than T. brucei gambiense, these parasites are morphologically indistinguishable. This is the probable reason why high sequence identity was displayed by other subspecies of the Brucei group. In addition, the high percent identity possessed by TbrOST II with other trypansomatids agrees with the evolutionarily conserved characteristics of the established OST. The DNA sequence data of TbrOST II showing similar sequences in the genome of other organisms further corroborate the previous reports regarding the ubiquitous nature of OST in other life forms. Based on the size of the amplicon and significant percentage of nucleotide and amino acid sequence identity to homologues within the genome of related species and various organisms, the results strongly indicate that TbrOST II is a trypanosome oligosaccharyl transferase gene candidate that should be fully characterized and subjected to functional genomic studies. The study reports the molecular cloning and sequencing of a potential oligosaccharyl transferase gene in $T$. brucei rhodesiense (TbrOST II). The sequence data has been deposited in the GenBank with accession number GU475126.
\end{abstract}

Keywords: Trypanosoma brucei rhodesiense, putative oligosaccharyl transferase, N-glycosylation. 
Baticados W.N., Inoue N., Sugimoto C., Nagasawa H. \& Baticados A.M. 2010. Genomic Cloning and Sequence Analysis of Trypanosoma brucei rhodesiense Gene Encoding Putative N-glycosylation... Acta Scientiae Veterinariae. 38(3): 257-264.

\section{INTRODUCTION}

Trypanosoma brucei rhodesiense is a haemoflagellate parasite of zoonotic significance. A wide range of mammalian fauna, especially domestic livestock and wild bovids, serves as reservoir host [23,26]. Aside from its public health importance, this parasite subspecies gained notoriety because of their effective system to circumvent the immune response of vertebrate host. The parasite cell surface is covered with millions of VSG dimers, which serve as an almost infinite repertoire of biomolecules needed for evasion of host immune system. Unique VSG are alternately produced by sequential expression of about a thousand trypanosome VSG gene reservoir per parasite; a phenomenon described as antigenic variation [5,6]. Around two decades ago, it was resolved that all trypanosome VSG is associated with one or more $\mathrm{N}$-linked oligosaccharides, with a range of structures including high mannose and complex types $[7,19,21,24]$. This complex process of protein modification is generally known as glycosylations, one of which is N-linked glycosylation.

The central event in the N-linked glycosylation process is catalyzed by oligosaccharyl transferase (OST) [11]. It catalyzes the co-translational addition of preassembled oligosaccharide complexes (Dol-PP$\mathrm{GlcNAc}_{2} \mathrm{Man}_{9} \mathrm{Glc}_{3}$ ) to an asparagine residue in an AsnXaa-Ser/Thr consensus sequon (Xaa can be any amino acid excluding proline) of the growing nascent polypeptide chain being translocated into the endoplasmic reticulum through a structure called translocon [4].

OST appears ubiquitous among eukaryotes and conserved throughout eukaryotic evolution. OST has been molecularly isolated and purified from mammalian sources, avian species and yeast Saccharomyces cerevisiae [3,11-14,22] as a multimeric enzyme.

In general, the incorporation of glycan structures to different protein moieties is precedent towards proper protein folding and stability, intracellular targeting, intercellular recognition, hormone synthesis, anti-apoptotic response, control of salt/osmotic stress, and cell surface expression of some glycoproteins $[8,9,15,17]$. Glycans can alter protein's antigenic properties and recently it was established that, glycan molecules associated with VSG were found to be important in several aspects of trypanosome-host interaction, especially during parasite evasion of the host defense mechanisms $[20,21,16]$. Therefore our major interest is to clone and characterize the trypanosome OST.
MATERIALSAND METHODS

\section{Laboratory animals}

Female 8-week-old BALB/c mice ${ }^{1}$ were used in the study. The animal room was maintained at $22 \pm 3^{\circ} \mathrm{C}$ with a 12:12 hours of light-dark cycle. All experiments were conducted according to the guidelines for the care and use of laboratory animals, Obihiro University of Agriculture and Veterinary Medicine, Japan.

\section{Trypanosomes and cultivation}

Trypanosoma brucei rhodesiense IL2343 strain (Ivory Coast) stabilates were maintained in BALB/c mice. After reaching high parasitemia ( 100 BSFs/field at magnification of $x 400$ ), infected blood was collected by intracardiac puncture, cultured and maintained in vitro in HMI-9 medium supplemented with $10 \%$ fetal bovine serum $^{2}$ as previously described [10]. The HMI-9 in vitro cultivation medium was prepared using the following composition: Iscove's modified dulbecco's medium ${ }^{3}$ (IMDM), $10 \mathrm{mM}$ bathocuproine disulfonic $\mathrm{acid}^{3}, 100$ $\mathrm{mM}$ pyruvic acid sodium salt ${ }^{3}, 16 \mathrm{mM}$ thymidine ${ }^{3}, 40$ $\mathrm{mg} / \mathrm{mL}$ bovine serum albumin ${ }^{3}, 1 \mathrm{mg} / \mathrm{mL}$ bovine holotransferrin, $100 \mathrm{mM}$ hypoxanthine $\mathrm{e}^{4}, 150 \mathrm{mM}$ L-cysteine hydro-chloride $^{3}, 14 \mathrm{mM}$ 2-mercaptoethanol ${ }^{5}, 25 \mathrm{mM}$ HE-PES $^{3}$ pH 7.2 , 200 mM L-glutamine ${ }^{6}, 10 \% \mathrm{v} / \mathrm{v}$ heat inactivated fetal bovine serum ${ }^{2}, 100 \mathrm{U} / \mathrm{mL}$ penicillin ${ }^{7}$ and $100 \mu \mathrm{g} / \mathrm{mL}$ streptomycin ${ }^{7}$.

\section{DNA extraction}

The template genomic DNA for PCR amplification was extracted as described previously [2]. Briefly, T. brucei rhodesiense IL2343 genomic DNA was extracted by adding 9 volumes of extraction buffer $\left(0.2 \mathrm{M} \mathrm{NaCl}^{8}, 10 \mathrm{mM}\right.$ Tris- $\mathrm{HCl}^{3,8} \mathrm{pH} 8.0,10 \mathrm{mM}$ EDTA $^{3} \mathrm{pH} 8.0$ and $1 \%$ SDS), proteinase $\mathrm{K}^{6}$ to a final concentration of $100 \mu \mathrm{g} / \mathrm{mL}$ and followed by 6 hours incubation at $55^{\circ} \mathrm{C}$ with gentle agitation. Overnight incubation was performed after additional proteinase $\mathrm{K}$ was placed. Genomic DNAs were phenolchloroform-isoamyl alcohol ${ }^{3}$ extracted, ethanol precipitated, and resuspended in Tris-EDTA buffer, $\mathrm{pH}$ 8.0 or deionized water. The concentration of the sample DNA was determined by spectrophotometry.

\section{Polymerase chain reaction amplification of putative $O S T$ gene}

The primers were designed from the nucleotide sequences of $T$. brucei genomic clones as guided by EMBL-EBI Parasite Genomes WU-Blast 
2 database search (www.ebi.ac.uk/blast/parasites.html) for African trypanosomes with L. major putative OST STT3 subunit sequence as the query [EMBL Q9U5N8 (AJ251127.1)]. PCR amplication was performed using a forward primer' ${ }^{9}\left(5^{\prime}-\mathrm{TGG}\right.$ TAC GAC TAC ATG AGC TGG TAC CCG CT-3') and a reverse primer' ${ }^{9}$ (5'-TGG ATC TCC TTC GCT GGC GGG TAC TG-3'). Distilled water was used as template for negative control reaction. The samples were programmed to a temperature-step cycle of $94^{\circ} \mathrm{C}$ at $10 \mathrm{~min}, 94^{\circ} \mathrm{C}$ at $30 \mathrm{sec}, 60^{\circ} \mathrm{C}$ at $30 \mathrm{sec}$ for a total of 30 cycles followed by $4 \mathrm{~min}$ extension at $72^{\circ} \mathrm{C}$. The PCR products were analyzed by electrophoresis on 1\% TAE (Tris-acetate-EDTA) agarose gel. The PCR product was then processed for cloning after agarose gel extraction using a commercial $\mathrm{kit}^{10}$ according to the manufacturer's instructions.

\section{Cloning and sequencing of PCR products}

The PCR product was ligated into EcoR V site of pT7 bluescript plasmid vector ${ }^{11}$ using Takara solution I ligation $\mathrm{kit}^{12}$. Ligation reaction was transformed into DH5 $\alpha$ competent E.coli cells and plated on Luria Britani's-ampicillin (LB-amp) agar dishes. The presence of insert was confirmed by restriction digest against Hind III \& Xba I site from the cloning site of the plasmid vectors flanking the PCR product.

Prior to sequencing, twenty-five cycles of Bigdye PCR was carried out in a total volume of $5 \mu \mathrm{l}$ and were performed using the following standard condition: $96^{\circ} \mathrm{C}$ at $2 \mathrm{~min}, 96^{\circ} \mathrm{C}$ at $10 \mathrm{sec}, 50^{\circ} \mathrm{C}$ at 5 sec and $60^{\circ} \mathrm{C}$ at 4 minutes. Sequencing was started by the single strand dideoxynucleotide-chaintermination method using a cycle sequencing kit $^{13}$, DNA sequence analyzer ${ }^{13}$ and $\mathrm{T} 7$ promoter primer and pUC/M13 reverse primers ${ }^{9}$. The second set ${ }^{9}$ [forward (5'-GAC ATA CAG CGT CAG TTT GC3'); reverse (5'-GAT GAA TGT GAG TGA AGA GAG C-3')] and the third set ${ }^{9}$ [forward (5'-CGT TCG GAT TCT TCA AAC CTA CAG-3') and reverse (5'AAT ACG GGC ATC TTC AGG CG-3')] primers were used to obtain the partial nucleotide sequence. The nucleotide sequence alignments and analysis were performed using sequence analysis softwares ${ }^{14,15}$.

\section{RESULTS}

A putative trypanosome oligosaccharyl transferase gene was successfully amplified using $T$. brucei rhodesiense crude DNA as the template.
Genomic clone of approximately 2000 bp was obtained after PCR amplification (Figure 1). Nucleotide sequencing revealed that the amplified band was composed of 2057 bp partial nucleotide sequence (Figure 2). The deduced partial primary structure of the T. brucei rhodesiense putative oligosaccharyl transferase clone II (TbrOST II) was composed of 685 amino acid sequence (Figure 2). Determination of the sequence homology in other organisms was carried out using NCBI Basic Local Alignment Search Tool (BLAST) (http://blast.ncbi.nlm.nih.gov/Blast.cgi) [1].

Sequence analysis showed that TbrOST II had significant nucleotide (Ns) and amino acid sequence (As) percent identity to putative oligosaccharyl transferase subunit coming from other kinetoplastid genome in the NCBI public database.

When compared with the related trypanosome species, the DNA size of T. brucei rhodesiense putative oligosaccharyl transferase was $200 \mathrm{bp}$ than $T$. brucei gambiense (1842 bp, FN554968.1). In addition, the sequence homologues from T. brucei (2466 bp, XM_839672.1) (2466 bp, XM_839671.1) (2406 bp, AC159432.1)(2406 bp, XM_839670.1), exceeded TbrOST II clone by $>400$ bp. The Latin American trypanosome species, T. cruzi, with percent identity to TbrOST II on the other hand had a molecular weight of 2397 bp. (XM_803446.1). Furthermore, the data showed that the annotated DNA size of the homologues under genus Leishmania was greater than that of genus Trypanosoma with

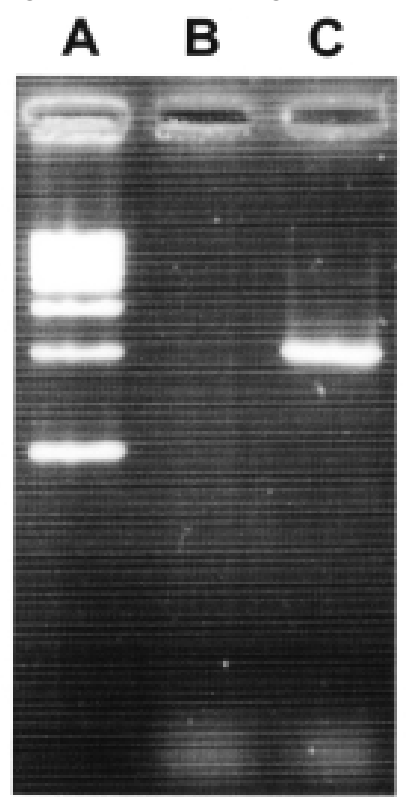

Figure 1. Polymerase Chain Reaction amplification of Trypanosoma brucei rhodesiense Putative Oligosaccharyl transferase clone II ( TbrOST II). Lane (A), (B) \& (C) represents $1 \mathrm{kbp}$ DNA ladder, negative control reaction and $\sim 2,000$ bp PCR amplicon respectively. 
a disparity ranging from $\sim 300-500 \mathrm{bp}$ (L. infantum) and 200- 500bp (L. major \& L. braziliensis).

Leishmania infantum displayed the greatest difference in terms of DNA molecular weight to TbrOST II (2592 bp, XM_001468890.1) (2385 bp, XM_001468892.1)(2355 bp, XM_001468891.1) followed by L. braziliensis (2580 bp, XM_0015 65753.1) (2319 bp, XM_001568165.1) (2565 bp, XM_001568167.1) (2472 bp, XM_001568166.1) and the least was L. major (2574 bp, XM_838130.1) (2322 bp, XM_838128.1) (2559 bp, XM_838127.1) (2373 bp, XM_838129.1)(2502 bp, AJ251127.1).

The molecular size of the amplicon showed akin to several homologues under order kinetoplastida. Taking into consideration the reported DNA size of the homologues from closely related species, the data suggest that the full-length nucleotide sequence of $T$. brucei rhodesiense putative oligosaccharyl transferase is also not more than $3000 \mathrm{bp}$.

Furthermore, the results using BLAST nucleotide (Ns) and amino acid (As) homology search showed that the $T$. brucei rhodesiense putative oligosaccharyl transferase had a very high percent sequence identity to the genome of $T$. brucei (Ns:98\%,XM_839670.1; As:98\%, XP_844763.1). Other homologues found in the genome of $T$. brucei (Ns:95\%, XM_839672.1; As:78\%, XP_844765.1) (Ns:96\%, XM_839671.1; As:77\%, XP_844764.1) and T. brucei gambiense (Ns:99\%, FN554968.1; As:78\%, CBH10989.1) also showed very high DNA sequence identity but with only fairly high amino acid sequence identity to TbrOST II. A moderate sequence similarity in the genome of T. cruzi (Ns:74\%, XM_803446.1; As:63\%, XP_808539.1) was also observed.

Subsequently, percent sequence identity hits in other members of family Trypanosomatidae, genus Leishmania, were also obtained (Figure 3). All the homologues from genus Leishmania displayed moderate and low nucleotide and amino acid sequence identity to TbrOST II respectively. The following are the different Leishmania clones from the GenBank with nucleotide and amino acid

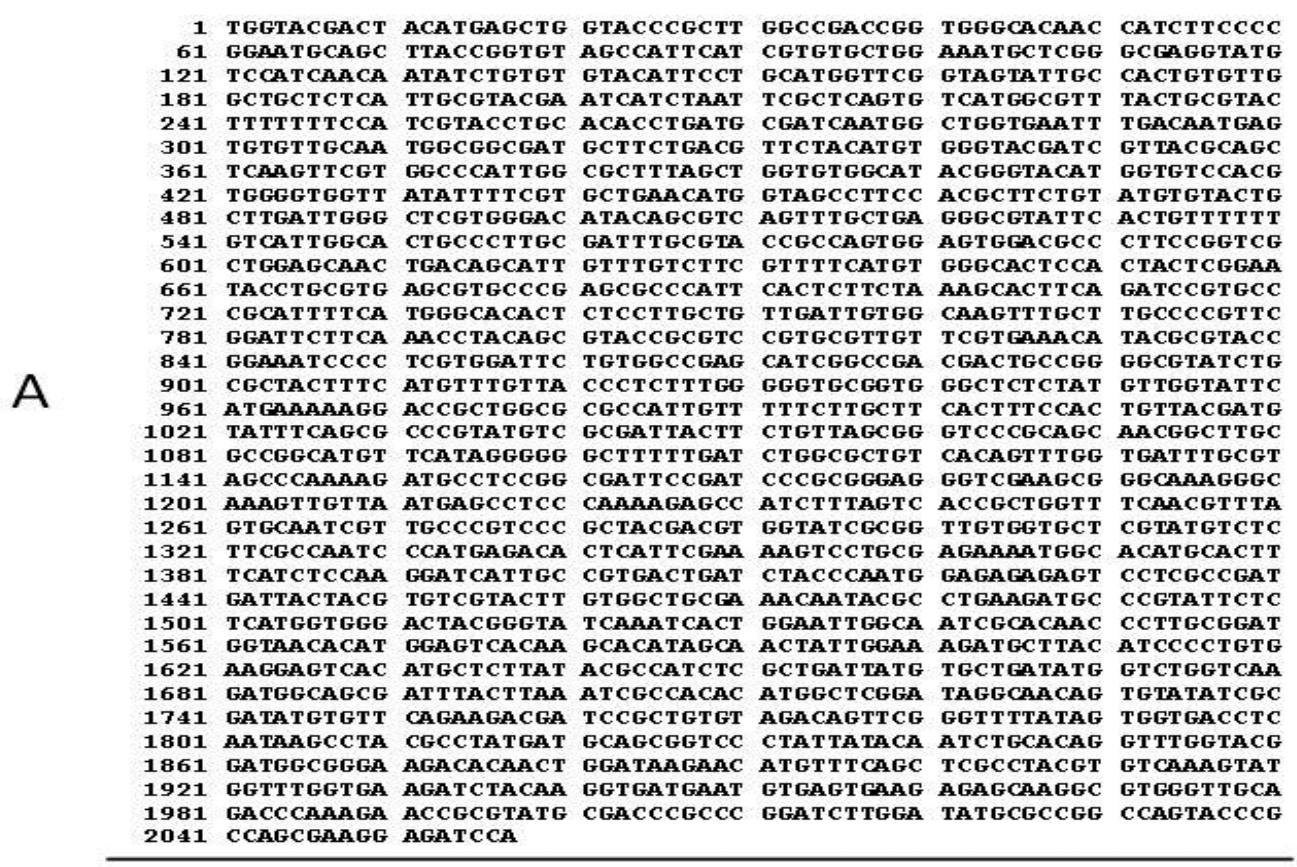

1 WYDYMSWYPL GRPVGTTIFP GMOLTGVATH RVLEMLGRG SINHICVYIP AWFGSIATVL 61 AALIAYESSH SLSUMAFTAY FFSTVPAHWM RSMAGEFDHE CVAMAMMLLT FYMTVRSLRS 121 SSSWPIGALA GVAYGYMVST WGGYIFVLM VAFHASVCVL LDHARGTYSV SLLRAYSLFF 181 VIGTALAICV PPVEWTPFRS LEQLTALFVF VFMHALHYSE YLRERARAPI HSSKALQIRA 181 RIGTALALS PTEL LTVALLAPF GFFKPTAYR RALFVKHTRT GHPLVDSVAE HRPTTAGAYL 241 RIFMGTLSLL LTVASLLAPF GFFKPTAYTN RALFVKHTRT GNPLVDSVAE HRPTTAGAYL
301 RYFHVCYPLW GCGGLSMIVF MKKDRHRAT FLASLSTVTM YF SAFASRLL LLAGPAATAC

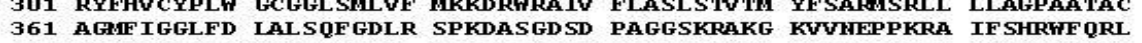
421 VQSLPVPLRR GIAVNLVCL FAHPMRHSFE KSCEKMAHAL SSPRIIAVTD LPHGERVLAD 481 DYYVSYLWLR MWTPEDARIL SWWYGYQIT GIGHRTTLAD GHTWSHKHIA TIGKALTSPV 541 KESHALIRHL ADYVLIWSGQ DGSDLLKSPH MARIGHSYYR DMCSEDDPLC RQFGFYSGDL 601 WKPTPMMORS LLYHLHRFGT DGGKTQLDKA MFQLAYVSKY GLVKTYKVMT VSEESKAHVA 661 DPKHRVCDPP GSWICAGQYP PAKEI

Figure 2. Partial Nucleotide (A) and Deduced Amino Acid (A) Sequence of Trypanosoma brucei rhodesiense Putative Oligosaccharyl transferase (TbrOST II) (GenBank: GU475126). 
Baticados W.N., Inoue N., Sugimoto C., Nagasawa H. \& Baticados A.M. 2010. Genomic Cloning and Sequence Analysis of Trypanosoma brucei rhodesiense Gene Encoding Putative N-glycosylation... Acta Scientiae Veterinariae. 38(3): 257-264.

sequenced regions having local similarity to TbrOST II: L. infantum (Ns:70\%, XM_001468891.1; As:57\%, XP_001468928.1) (Ns:70\%, XM_001468890.1; As:56\%, XP_001468927.1) (Ns:83\%, XM_001468 892.1; As:46\%, XP_001468929.1); L. braziliensis (Ns:69\%, XM_001565753.1; As:54\%, XP_00156 5803.1) (Ns:69\%, XM_001568165.1; 55\%,As:XP
_001568215.1) (Ns:70\%, XM_00156 8167.1; As:55\%, XP_001568217.1) (Ns:81\%, XM_0015681 66.1; As:46\%, XP_001568216.1); L. major (Ns:69\%, XM_838130.1; As:55\%, XP_843223.1) (Ns:70\%, XM_838128.1;As:57\%, XP_843221.1) (Ns:70\%, XM_838127.1; As:55\%, XP_843220.1) (Ns:80\%, XM_838129.1; As:46\%, XP_843222.1) (Ns:80\%,

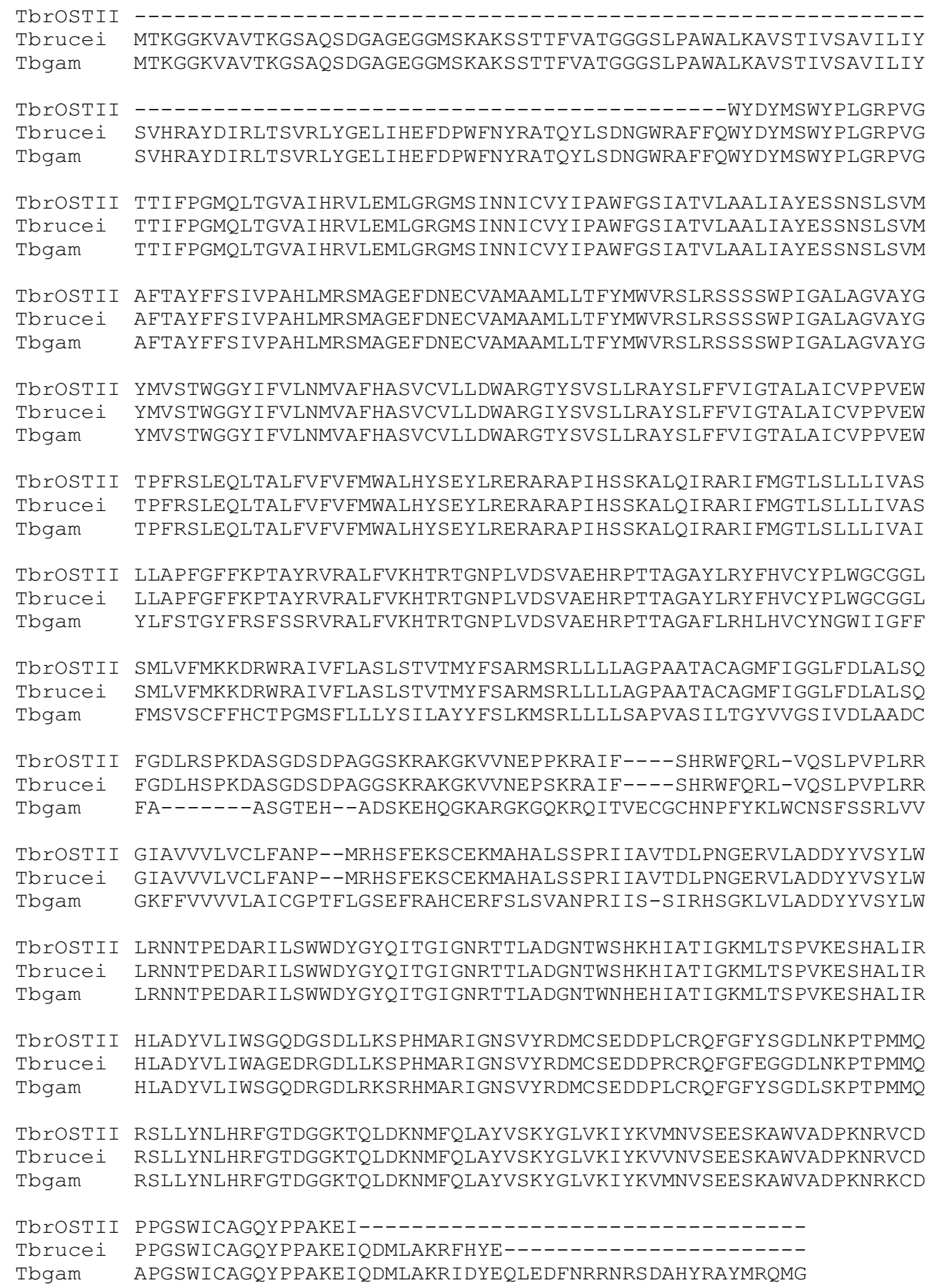

Figure 3. Amino Acid sequence identity of Trypanosoma brucei rhodesiense Putative Oligosaccharyl transferase (TbrOST II) within genome of genus Trypanosoma, family Trypanosomatidae. Alinged sequences were from $T$. brucei -Tbrucei (98\%, XP_844763.1) and T. brucei gambiense -Tbgam (78\%, CBH10989.1) species. 
Baticados W.N., Inoue N., Sugimoto C., Nagasawa H. \& Baticados A.M. 2010. Genomic Cloning and Sequence Analysis of Trypanosoma brucei rhodesiense Gene Encoding Putative N-glycosylation... Acta Scientiae Veterinariae. 38(3): 257-264.

AJ251127.1; As:46\% , CAB61 569.1).

\section{DISCUSSION}

The surface coat of trypanosome species were previously reported to have marked diversities in $\mathrm{N}$ glycosylation [20]. This prompted us to hypothesize that trypanosomes also possess $\mathrm{N}$-glycosylation enzyme. During the attempt to clone T. brucei rhodesiense putative oligosaccharyl transferase, a genomic clone of $\sim 2000$ bp was acquired.

Notably, L. major putative OST STT3 subunit sequence was used as the query during the primer design for PCR amplification of TbrOST II. The efficient amplification of the gene in genus T. brucei rhodesiense using primers designed from L. major stt 3 gene as the query strongly indicate that TbrOST II seems to be conserved within the family Trypanosomatidae. The nucleotide BLAST results indicates that sequence identity is higher between species of the same genus rather than of the same family. It is known that T. brucei, T. gambiense and $T$. rhodesiense are members of the Brucei-complex or Brucei group. Although T. brucei brucei has more similarities with $T$. brucei rhodesiense than T. brucei gambiense, these parasites are morphologically indistinguishable [18,23]. This is the probable reason why high sequence identity was displayed by other subspecies of the Brucei group. This also indicates that the functional unit of this putative oligosaccharyl transferase is conserved within the Brucei complex trypanosomes. In addition, the high percent identity possessed by TbrOST II with other trypansomatids agrees with the evolutionarily conserved characteristics of the established OST [27, 25]. Consequently, DNA identity searches within the public databases obtained homologues in the genome of mammalian, nematode, arthropod and algae species. Selected organisms include Mus musculus (Ns:71\%, NM_ 024222.2; As:29\%, NP_077184.2), Schistosoma mansoni (Ns:73\%, XM_002577919.1; As:29\%, XP_002577965.12, Drosophila pseudoobscura pseudoobscura (Ns:77\%, XM_002134519.1; As:31\%, XP_002134555.1), Thalassiosira pseudonana (Ns:77\%, XM_002288187.1; As:46\%, XP_002288223.1) and Phaeodactylum tricornutum (Ns:73\%, XM_002185331.1; As:46\%, XP_002185367.1). Moreover, obtained amino acid sequence identity in the genome of fly, nematode, mouse and algae homologues also exhibited lower sequence similarity (29-46\%) than the previously documented percent identity $(\sim 50 \%)$ of reported oligosaccharyl transferase in other eukaryotic species [25].

\section{CONCLUSIONS}

Finally, even currently regarded as putative, DNA sequence data of TbrOST II showing similar sequences in the genome of other organisms further corroborate the previous reports regarding the ubiquitous nature of OST in other life forms. Based on the size of the amplicon and significant percentage of nucleotide and amino acid sequence identity to homologues within the genome of related species and various organisms, the results strongly indicate that TbrOST II is a trypanosome oligosaccharyl transferase gene candidate that should be fully characterized and subjected to functional genomic studies. The study reports the molecular cloning and sequencing of a potential oligosaccharyl transferase gene in T. brucei rhodesiense (TbrOST II). The sequence data has been deposited in the GenBank with accession number of GU475126.

\section{SOURCES AND MANUFACTURERS}

${ }^{1}$ CLEA Japan, Inc., Tokyo, Japan.

${ }^{2}$ Biosource International Inc., Camarillo, CA, USA.

${ }^{3}$ Sigma, St. Louis, USA.

${ }^{4}$ Calbiochem, La Jolla, CA, USA.

${ }^{5} \mathrm{BDH}$ Chemicals, Poole, England.

${ }^{6}$ Invitrogen Co., Carlsbad, CA, USA.

${ }^{7}$ Meiji Seika Kaisha, Ltd., Tokyo, Japan.

${ }^{8}$ Wako Pure Chemical Industries Ltd., Osaka, Japan.

${ }^{9}$ Sigma-Genosys, Hokkaido, Japan.

${ }^{10}$ GeneClean kit II, Q Biogene, Vista, CA, USA.

${ }^{11}$ Novagen, Madison, WI, USA.

${ }^{12}$ TAKARA Shuzo, Japan.

${ }^{13}$ Applied Biosystems, Forster City, CA, USA.

${ }^{14} \mathrm{Mac}$ Vector 6.5 sequence analysis software, Oxford University, England.

${ }^{15}$ CLC Workbench 5.6 software. USA.

Acknowledgment. This work was supported by the Japanese Ministry of Education, Culture, Sports, Science and Technology (Monbukagakusho). 
Baticados W.N., Inoue N., Sugimoto C., Nagasawa H. \& Baticados A.M. 2010. Genomic Cloning and Sequence Analysis of Trypanosoma brucei rhodesiense Gene Encoding Putative N-glycosylation... Acta Scientiae Veterinariae. 38(3): 257-264.

\section{REFERENCES}

1 Altschul S.F., Madden T.L., Schäffer A.A., Zhang J., Zhang Z., Miller W. \& Lipman D.J. 1997. "Gapped BLAST and PSIBLAST: a new generation of protein database search programs". Nucleic Acids Research. 25(17): 3389-3402.

2 Baticados W.N., Witola W.H., Inoue N., Kim J., Kuboki N., Xuan X., Yokoyama N. \& Sugimoto C. 2005. Expression of a Gene Encoding Trypanosoma congolense Putative Abc1 Family Protein is Developmentally Regulated. Journal of Veterinary Medical Science. 67(2): 157-164.

3 Breuer W. \& Bause E. 1995. Oligosaccharyl transferase is a constitutive component of an oligomeric protein complex from pig liver endoplasmic reticulum. European Journal of Biochemistry. 228(3): 689-696.

4 Brodsky J.L. 1997. Translocation of proteins across the endoplasmic reticulum membrane. International Review of Cytology. 178: 277-328.

5 Cross G.A.M. 2002. Antigenic variation in African trypanosomes and malaria. In: Molecular Medical Parasitology. Marr J.J., Nilsen T.W. \& Komuniecki R.W. (Eds). Academic Press: New York, pp.89-110.

6 Donelson J.E., Hill K.L. \& El-Sayed N.M.A. 1998. Multiple mechanism of immune evasion by African trypanosomes. Molecular \& Biochemical Parasitology. 91(1): 51-66.

7 Ferguson M.A.J. 1997. The structure and biosynthesis of trypanosomatid glycosylphosphatidylinositols. In: Hide G. \& Mottram J.C. (Eds). Trypanosomiasis and Leishmaniasis. U.K.: Commonwealth Agricultural Bureaux International, pp.65-77.

8Garcia R.C., Cundell D.R., Tuomanen E.I., Kolakowski Jr. L.F., Gerard C. \& Gerard N.P. 1995. The role of N-glycosylation for functional expression of the human platelet-activating factor receptor. Glycosylation is required for efficient membrane trafficking. Journal of Biological Chemistry. 270(11): 25178-25184.

9 Goder V., Bieri C. \& Spiess M. 1999. Glycosylation can influence topogenesis of membrane proteins and reveals dynamic reorientation of nascent polypeptides within the translocon. Journal of Cell Biology. 147(2): 257-265.

10 Hirumi H. \& Hirumi K. 1991. In vitro cultivation of Trypanosoma congolense bloodstream forms in the absence of feeder cell layers. Parasitology. 102(2): 225-236.

11 Kaplan H.A., Welply J.K. \& Lennarz W.J. 1987. Oligosaccharyl transferase: the central enzyme in the pathway of glycoprotein assembly. Biochimica et Biophysica Acta. 906(2): 161-173.

12 Kelleher D.J. \& Gilmore R. 1997. DAD1, the defender against apoptotic cell death, is a subunit of the mammalian oligosaccharyltransferase. Proceedings of the National Academy of Sciences. 94(10): 4994-4999.

13 Knauer R. \& Lehle L. 1999. The oligosaccharyltransferase complex from Saccharomyces cerevisiae. Journal of Biological Chemistry. 274 (24): 17249-17256.

14Kumar V., Heinemann F.S. \& Ozols J. 1994. Purification and characterization of avian oligosaccharyltransferase. Complete amino acid sequence of the 50-kDa subunit. Journal of Biological Chemistry. 269(18): 13451-13457.

15Levin D. \& Bartlett-Heubusch E. 1992. Mutants in the S. cerevisiae PKC1 gene display a cell cycle-specific osmotic stability defect. Journal of Cell Biology. 116(5): 1221-1229.

16Lisowska E. 2002. The role of glycosylation in protein antigenic properties. Cellular and Molecular Life Sciences. 59(3): 445-455.

17 Live D.H., Kumar R.A., Beebe X. \& Danishefsky S.J. 1996. Conformational influences of glycosylation of a peptide: A possible model for the effect of glycosylation on the rate of protein folding. Proceedings of the National Academy of Sciences. 93(23): 12759-12761.

18 Matthews K.R. 2005. The developmental cell biology of Trypanosoma brucei. Journal of Cell Science. 118(2): 283-290.

19 Mehlert A., Bond C.S. \& Ferguson M.A.J. 2002. The glycoforms of a Trypanosoma brucei variant surface glycoprotein and molecular modeling of glycosylated surface coat. Glycobiology. 12(10): 607-612.

20 Parodi A.J. 1993. N-glycosylation in trypanosomatid protozoa. Glycobiology. 3(3): 193-199.

21Pays E. \& Nolan D.P. 1998. Expression and function of surface proteins in Trypanosoma brucei. Molecular \& Biochemical Parasitology. 91(1): 3-36.

22 Silberstein S., Kelleher D.J. \& Gilmore R. 1992. The 48-kDA subunit of the mammalian oligosaccharyltransferase complex is homologous to the essential yeast protein WBP1. Journal of Biological Chemistry. 267(33): 23658-23663.

23 Soulsby E.J.L. 1982. Phylum: Sarcomastigophora Honigberg and Balamuth, 1963, Subphylum: Mastigophora Diesing, 1866. In: Soulsby E.J.L. (Ed). Helminths, Arthropods and Protozoa of Domesticated Animals. 7th edn. London: Baillière Tindall, pp.514-582.

24 Strickler J.E. \& Patton C.L. 1980. Trypanosoma brucei brucei: Inhibition of glycosylation of the major variable surface coat glycoprotein by tunicamycin. Proceedings of the National Academy of Sciences. 77(3): 1529-1533. 
Baticados W.N., Inoue N., Sugimoto C., Nagasawa H. \& Baticados A.M. 2010. Genomic Cloning and Sequence Analysis of Trypanosoma brucei rhodesiense Gene Encoding Putative N-glycosylation... Acta Scientiae Veterinariae. 38(3): 257-264.

25 Yan Q. \& Lennarz W.J. 2002. Studies on the function of oligosaccharyl transferase subunits. Journal of Biological Chemistry. 277(49): 47692-47700.

26 Zoller T., Fèvre E.M., Welburn S.C., Odiit M. \& Coleman P.G. 2008. Analysis of risk factors for T. brucei rhodesiense sleeping sickness within villages in south-east Uganda. BMC Infectious Diseases. 8(88): 1-9.

27 Zufferey R., Knauer R., Burda P., Stagljar I., Heesen S., Lehle L. \& Aebi M. 1995. STT3, a highly conserved protein required for yeast oligosaccharyl transferase activity in vivo. EMBO Journal. 14(20): 4949-4960. 